\title{
Capacidade erro-zero de canais quânticos com medições coletivas
}

\author{
Rex A. C. Medeiros ${ }^{\dagger \S}$, Francisco M. de Assis $^{\S}$, Romain Alléaume ${ }^{\dagger}$ e Gérard Cohen ${ }^{\dagger}$
}

\begin{abstract}
Resumo-A capacidade erro-zero de um canal quântico foi definida como sendo o supremo das taxas em que informação clássica pode ser transmitida através de um canal quântico, considerando uma probabilidade de erro igual a zero. Neste artigo, o valor da capacidade é analisado para o caso em que medições entrelaçadas são permitidas na saída do canal. É mostrado que medições coletivas podem aumentar a capacidade erro-zero dos canais quânticos. É também mostrado um exemplo de um canal quântico para o qual a capacidade erro-zero é alcançada usando uma família de estados quânticos não ortogonais na entrada do canal.
\end{abstract}

Palavras-Chave-Canais quânticos, capacidade erro-zero, medições entrelaçadas.

Abstract-The zero-error capacity of a quantum channel was defined as the least upper bound of rates at which classical information can be transmitted without error through a noisy quantum channel. This paper investigates the behavior of the quantum zero-error capacity when entangled measurements between several channel outputs are allowed. It is shown that collective measurements may increase the channel capacity. We also show an example of a quantum channel for which the zeroerror capacity is reached using an ensemble of non-orthogonal input states.

Keywords-Quantum channels, zero-error capacity, collective measurements.

\section{INTRODUÇão}

A teoria da informação quântica, assim como a teoria da informação clássica, geralmente propõe soluções assintóticas para o problema da determinação da capacidade de canais, em que uma probabilidade de erro arbitrariamente pequena existe mesmo quando se faz uso do melhor esquema de codificação. Ao que concerne a transmissão de informação clássica através de um canal quântico, se encaixam nesta categoria:

1) a capacidade de Holevo-Schumacher-Westrmoreland (HSW), definida como a taxa assintótica máxima na qual a informação clássica pode ser transmitida confiavelmente, usando codificação e decodificação quânticas [1], [2];

2) a capacidade auxiliada por entrelaçamento $C_{E}$, que é o supremo das taxas para transmissão de informação clássica através de um canal quântico quando uma quantidade ilimitada de entrelaçamento está disponível entre o transmissor e o receptor [3];

\footnotetext{
† Département Informatique et Réseaux, École Nationale Supérieure des Télécommunications, Paris, France

$\S$ Departamento de Engenharia Elétrica, Universidade Federal de Campina Grande, Campina Grande, Brazil.

Emails: medeiros@enst.fr, fmarcos@dee.ufcg.edu.br, alleaume@enst.fre cohen@enst.fr
}

3) a capacidade adaptativa de Shor [4], em que o sistema de medição dos estados quânticos na saída do canal pode variar segundo o resultado de medições passadas.

Nos casos 1) e 2) o protocolo de comunicação restringe à entrada do canal a produtos tensoriais de estados quânticos, enquanto que as medições na saída são entrelaçadas entre vários usos do canal. A capacidade de Shor prevê medições individuais para cada estado quântico recebido.

Recentemente, Medeiros e Assis [5] generalizaram a capacidade erro-zero de canais clássicos discretos sem memória [6], [7] para canais quânticos. A capacidade erro-zero quântica (CEZQ) foi definida como o supremo das taxas para a transmissão de informação clássica através de um canal quântico ruidoso, com a restrição de que a probabilidade de erro deve ser igual a zero. Quanto ao protocolo, as entradas são restritas a produtos tensoriais e as medições são tomadas individualmente na saída do canal. Vários foram os desenvolvimentos feitos em torno da CEZQ [8], [9]. Em particular, foi demonstrado que a capacidade HSW é um limitante superior para a CEZQ [10], bem como conexões com a teoria de subsistemas quânticos sem ruído e subespaços livres de decoerência[11].

Neste trabalho será analisado o que acontece com o valor da capacidade erro-zero quântica quando são permitidas medições entrelaçadas entre várias saídas do canal. Como no caso da capacidade HSW, é mostrado que tais medições podem contribuir para aumentar o valor da capacidade erro-zero de canais quânticos. Nesta abordagem, uma maximização sobre as medições (POVM) torna-se desnecessária, contrariamente ao caso em que a capacidade erro-zero é calculada considerando medições individuais [12]. Por último, será mostrado um exemplo de um canal quântico tal que sua CEZQ é alcançada usando um subconjunto de estados quânticos nãoortogonais na entrada do canal. Ainda, este canal quântico dá origem ao pentágono como grafo característico, de forma que a CEZQ pode ser precisamente calculada e um código atingindo a capacidade explicitado.

$\mathrm{O}$ artigo está organizado como segue. A Seç. II apresenta um resumo da definição da capacidade erro-zero quântica, bem como desenvolvimentos necessários à compreensão do restante do artigo. As várias formas de definir capacidades erro-zero para a transmissão de informação clássica através de canais quânticos são discutidas na Seç. III. Na Seç. IV a capacidade erro-zero é analisada para o caso onde são consideradas medições coletivas na saída do canal. Finalmente, a Seç. V mostra um exemplo de um canal quântico cuja capacidade é alcançada para estados não-ortogonais na entrada do canal. As conclusões são tecidas na Seç. VI. 


\section{CAPACIDAdE ERRO-ZERO DE CANAIS QUÂNTICOS}

Com o objetivo de facilitar a leitura deste trabalho, será apresentada nesta seção um resumo da capacidade erro-zero de canais quânticos tal como ela foi definida em [5].

Seja $\mathcal{E}$ um canal quântico definido num espaço de Hilbert $\mathcal{H}$ de dimensão $d$. Tal canal quântico pode ser modelado por um operador linear, completamente positivo e que preserva o traço dos operadores de densidade na entrada do canal, $\mathcal{E} \equiv$ $\left\{E_{a}\right\}$, em que $E_{a}$ são operadores de Kraus em $\mathcal{H}$ satisfazendo $\sum_{a} E_{a}^{\dagger} E_{a}=\mathbb{1}$ [13]. A saída do canal para uma entrada $\rho_{i}$ é dada por

$$
\mathcal{E}\left(\rho_{i}\right)=\sum_{a} E_{a} \rho_{i} E_{a}^{\dagger}
$$

O canal quântico $\mathcal{E}$ é dito ser sem memória se o mesmo não produz entrelaçamento na saída quando produtos tensoriais de estados quânticos são colocados na entrada. Neste caso, $n$ usos do canal quântico para a transmissão de um estado $\bar{\rho}_{i}=\rho_{i_{1}} \otimes$ $\cdots \otimes \rho_{i_{n}}$ produz uma saída $\mathcal{E}\left(\bar{\rho}_{i}\right)$ que é dada por

$$
\begin{aligned}
\mathcal{E}\left(\bar{\rho}_{i}\right) & =\mathcal{E}\left(\rho_{i_{1}}\right) \otimes \cdots \otimes \mathcal{E}\left(\rho_{i_{n}}\right) \\
& =\bigotimes_{j=1}^{n} \mathcal{E}\left(\rho_{i_{j}}\right) .
\end{aligned}
$$

O protocolo de comunicação originalmente proposto pode ser resumido como segue. É definido um subconjunto finito de estados quânticos de entrada $\mathcal{S}=\left\{\rho_{1}, \ldots, \rho_{l}\right\}$, em que cada estado $\rho_{i} \in \mathcal{S}$ reside num espaço de Hilbert de entrada $\mathcal{H}_{E}$ de mesma dimensão que $\mathcal{H}$. As palavras-código de um código quântico de erro-zero de comprimento $n$ são produtos tensoriais de estados em $\mathcal{S}, \bar{\rho}_{i}=\rho_{i_{1}} \otimes \cdots \otimes \rho_{i_{n}}$, os quais são definidos num espaço produto $\mathcal{H}_{E}^{\otimes n}$ de dimensão $d^{n}$. Por sua vez, um código de bloco quântico de erro-zero e de comprimento $n$ é um mapeamento de $K_{n}$ mensagens clássicas (que podem ser representadas por índices $1, \ldots, K_{n}$ ) em um subconjunto de palavras-código de comprimento $n$. Claramente, a taxa deste código é dada por $R=\frac{1}{n} \log K_{n}$.

$\mathrm{Na}$ recepção, as seqüências recebidas são medidas usando um POVM (Positive Operator-Valued Measurements), $\mathcal{P}=$ $\left\{M_{1}, \ldots, M_{m}\right\}$, em que $M_{i}$ são operadores no espaço de Hilbert de saída $\mathcal{H}_{S}$ de dimensão $d$. Isto implica que o protocolo emprega medições individuais, ou seja, cada estado $\mathcal{E}\left(\rho_{i_{j}}\right)$ da palavra-código de saída $\mathcal{E}\left(\bar{\rho}_{i}\right)=\mathcal{E}\left(\rho_{i_{1}}\right) \otimes \cdots \otimes$ $\mathcal{E}\left(\rho_{i_{n}}\right)$ é medido individualmente. Neste caso, a medição de $\mathcal{E}\left(\bar{\rho}_{i}\right)$ produz uma de palavra de saída, $w_{n} \in\{1, \ldots, m\}^{n}$.

$\mathrm{O}$ esquema de decodificação para um código de bloco quântico de comprimento $n$ é uma função que associa univocamente cada palavra de saída a inteiros de 1 a $K_{n}$ que representam as mensagens clássicas. A probabilidade de erro para este código é maior do que zero se o sistema de decodificação identifica na saída uma mensagem diferente daquela enviada.

A Fig. 1 ilustra o diagrama de blocos de um sistema de comunicações para a transmissão de mensagens clássicas através de um canal quântico usando o protocolo de erro-zero descrito. Inicialmente é escolhido um índice $i$ que representa uma das $K_{n}$ mensagens clássicas. Em seguida, o codificador mapeia $i$ numa palavra-código de comprimento $n$. A sequiência é então transmitida através de um canal quântico ruidoso. $\mathrm{Na}$ recepção, são realizadas medições individuais definidas pelo
POVM $\mathcal{P}$ composto por operadores em $\mathcal{H}_{S}$. De posse da palavra de saída $w_{n}$, o decodificador deve decidir, sem erro, em um dos índices $1, \ldots, K_{n}$ que representa a mensagem realmente transmitida.

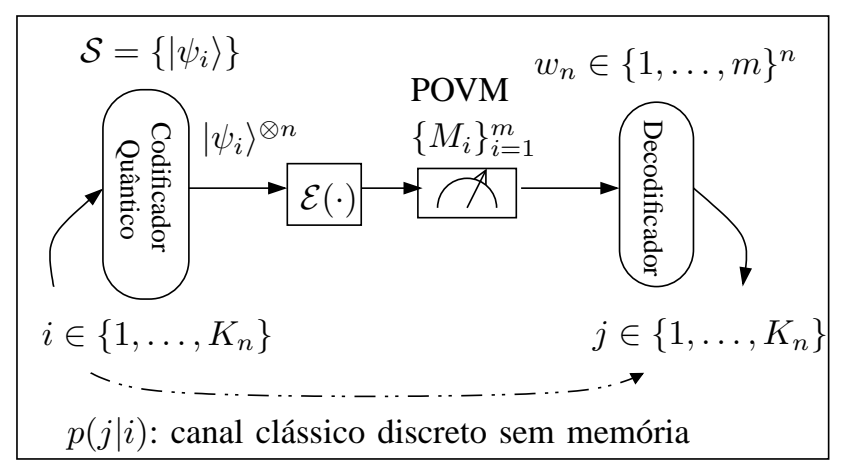

Fig. 1. Representação geral de um sistema de comunicações quântico para a transmissão de mensagem clássicas.

Originalmente, a capacidade erro-zero de um canal quântico foi definida como segue [5]:

Definição 1 Seja $\mathcal{E}$ um canal quântico representado por um operador linear, completamente positivo e que preserva $o$ traço. A capacidade erro-zero de $\mathcal{E}$, denotada por $C^{(0)}(\mathcal{E})$, é o supremo de todas as taxas alcançáveis com probabilidade de erro igual a zero. Isto é,

$$
C^{(0)}(\mathcal{E})=\sup _{n} \frac{1}{n} \log K_{n}
$$

em que $K_{n}$ é o número máximo de mensagens clássicas que o sistema pode transmitir sem erro, quando um código de bloco quântico de comprimento n é usado.

\section{A. Equivalente clássico}

A Definição 1 não faz menção sobre como a capacidade erro-zero de um canal quântico pode ser calculada. Medeiros e Assis [14] propuseram um procedimento geral para este cálculo, o qual é descrito a seguir.

Considere um canal quântico $\mathcal{E}$. Para uma escolha de um subconjunto $\mathcal{S}=\left\{\rho_{i}, \ldots, \rho_{l}\right\}$ de estados quânticos de entrada e de um POVM $\mathcal{P}=\left\{M_{1}, \ldots, M_{m}\right\}$, é possível definir um canal clássico discreto sem memória como segue:

- O alfabeto de entrada $X$ do canal discreto sem memória é composto pelos índices dos estados quânticos de entrada, ou seja, $X=\{1, \ldots, l\}$.

- O alfabeto de saída $Y$ é dado pelos índices dos operadores de medição, $Y=\{1, \ldots, m\}$.

- Os elementos da matriz estocástica do canal equivalente clássico são dados por

$$
p(y \mid x)=\operatorname{tr}\left[\mathcal{E}\left(\rho_{x}\right) M_{y}\right]
$$

Desta forma, para cada par $(\mathcal{S}, \mathcal{P})$ é possível definir um canal clássico discreto sem memória equivalente. Evidentemente, cada um desses canais equivalentes possui uma capacidade erro-zero (clássica) $C_{0}(\mathcal{S}, \mathcal{P})$. Foi mostrado [14], [5] que a capacidade erro-zero do canal quântico $\mathcal{E}$ é igual ao supremo 
das capacidades erro-zero clássicas $C_{0}(\mathcal{S}, \mathcal{P})$ sobre todos os pares $(\mathcal{S}, \mathcal{P})$ possíveis, ou seja,

$$
C^{(0)}(\mathcal{E})=\sup _{(\mathcal{S}, \mathcal{P})} C_{0}(\mathcal{S}, \mathcal{P})
$$

O par $(\mathcal{S}, \mathcal{P})$ que alcança o supremo na Eq. (5) é chamado de ótimo.

Para um canal quântico $\mathcal{E}$ e uma escolha de um par $(\mathcal{S}, \mathcal{P})$, dois estados $\rho_{i}, \rho_{j} \in \mathcal{S}$ são ditos ser adjacentes com relação ao POVM $\mathcal{P}$ se, e somente se, $\operatorname{tr}\left[\mathcal{E}\left(\rho_{i}\right) M_{k}\right]>0$ e $\operatorname{tr}\left[\mathcal{E}\left(\rho_{j}\right) M_{k}\right]>$ 0 para ao menos um $M_{k} \in \mathcal{P}$. Caso contrário, $\rho_{i}$ e $\rho_{j}$ são denominados não-adjacentes. É fácil verificar que se $\rho_{i}$ e $\rho_{j}$ são não-adjacentes com relação a $\mathcal{P}$, então é possível distinguir perfeitamente entre $\mathcal{E}\left(\rho_{i}\right)$ e $\mathcal{E}\left(\rho_{j}\right)$. Medeiros e Assis mostraram que um canal quântico possui capacidade erro-zero positiva se, e somente se, existir um $\operatorname{par}(\mathcal{S}, \mathcal{P})$ tal que pelo menos dois estados em $\mathcal{S}$ são não-adjacentes com relação ao POVM $\mathcal{P}[5]$.

Seguindo uma construção sugerida por Shannon [6], [7], o método de cálculo da capacidade erro-zero de canais quânticos via equivalente clássico pode ser reformulado usando elementos da teoria de grafos. Considere um canal quântico $\mathcal{E}$. Para um dado par $(\mathcal{S}, \mathcal{P})$ é possível construir um grafo característico $\mathcal{G}$ como segue. O conjunto de vértices é formado pelos índices de $\mathcal{S}, V(\mathcal{G})=\{1, \ldots, l\}$, e dois vértices $i, j \in$ $V(\mathcal{G})$ são conectados em $\mathcal{G}$ se os respectivos estados quânticos $\rho_{i}, \rho_{j} \in \mathcal{S}$ são não-adjacentes com relação ao POVM $\mathcal{P}$. A Eq. (5) pode ser então reescrita como [5], [11]:

$$
C^{(0)}(\mathcal{E})=\sup _{(\mathcal{S}, \mathcal{P})} \sup _{n} \frac{1}{n} \log \omega\left(\mathcal{G}^{n}\right),
$$

em que $\omega(\mathcal{G})$ é o número de cliques [15] do grafo $\mathcal{G}$, e $\mathcal{G}^{n}$ é o $n$-ésimo produto de $\mathcal{G}$ como definido em [7].

\section{FORMAS DE DEFINIR A CAPACIDADE ERRO-ZERO}

Como indicado na introdução, existem várias maneiras de definir capacidades de canais quânticos. Somente capacidades para transmissão de mensagens clássicas serão discutidas aqui. Com relação à capacidade erro-zero, existem quatro protocolos principais que podem originar valores diferentes de capacidades:

1) palavras-código são restritas a produtos tensoriais de estados quânticos de entrada, e medições são feitas individualmente na saída do canal;

2) entrelaçamento entre vários usos do canal é permitido, enquanto que medições são feitas individualmente na saída do canal;

3) palavras-código são restritas a produtos tensoriais de estados quânticos de entrada, enquanto que medições entrelaçadas entre várias saídas do canal são permitidas;

4) entrelaçamento entre vários usos do canal é permitido, como também medições entrelaçadas entre várias saídas.

A capacidade erro-zero na Def. 1 corresponde ao caso 1 ). Um dos problemas em aberto na teoria da informação é o da aditividade da capacidade HSW. Holevo conjeturou [2] que o uso de entrelaçamento na entrada não aumenta a capacidade
HSW de canais quânticos sem memória ${ }^{1}$. Embora não tenham formalmente provado, Medeiros e Assis [8] mostraram vários indícios de que a aditividade de Holevo também pode ser válida para a capacidade erro-zero.

Com relação às medições, é sabido que medidas entrelaçadas entre várias saídas do canal podem aumentar a informação mútua entre a entrada e a saída do canal quântico, sendo essenciais para atingir a capacidade HSW [13]. Desta forma, o protocolo do item 3) é de particular interesse e será discutido ao longo deste artigo. Será mostrado na próxima seção que o uso de medições entrelaçadas pode aumentar a capacidade $C^{(0)}(\mathcal{E})$ da Def. 1. Para este protocolo, será mostrado que não é necessária uma maximização sobre as medições $\mathcal{P}$, contrariamente ao caso da capacidade erro-zero com medições individuais, cujo valor é dado pelas Eqs. (5) e (6).

\section{CAPACIDADE ERRO-ZERO COM MEDIÇÕES COLETIVAS}

De maneira análoga à Sec. II, considere um canal quântico $\mathcal{E}$ num espaço de Hilbert $\mathcal{H}$ de dimensão $d$. Considere o protocolo do item 3) da Sec. III, descrito em detalhes abaixo:

- o alfabeto do código quântico de erro-zero é um subconjunto $\mathcal{S}=\left\{\rho_{1}, \ldots, \rho_{l}\right\}$ de estados quânticos pertencentes ao espaço de Hilbert de entrada $\mathcal{H}_{E}$ de dimensão $d$;

- para serem transmitidas através do canal quântico, as mensagens clássicas são mapeadas em palavras-código quânticas que são produtos tensoriais de estados em $\mathcal{S}$;

- são permitidas medições POVM entrelaçadas entre várias saídas do canal.

Antes de definir a capacidade, é necessário definir o esquema de (de)codificação:

Definição 2 Um código erro-zero de bloco $\left(K_{n}^{\infty}, n\right)$ para um canal quântico $\mathcal{E}$ é composto de:

1) um conjunto de índices $\left\{1, \ldots, K_{n}^{\infty}\right\}$, em que cada índice está associado a uma mensagem clássica;

2) uma função de codificação

$$
X^{n}:\left\{1, \ldots, K_{n}^{\infty}\right\} \rightarrow \mathcal{S}^{\otimes n},
$$

que mapeia mensagens clássicas em palavras-código $\bar{\rho}_{1}=X^{n}(1), \ldots, \bar{\rho}_{K_{n}^{\infty}}=X^{n}\left(K_{n}^{\infty}\right)$;

3) uma função de decodificação

$$
g:\{1, \ldots, m\} \rightarrow\left\{1, \ldots, K_{n}^{\infty}\right\},
$$

que associa, de maneira determinística, a cada saída $y \in\{1, \ldots, m\}$ de uma medição POVM $\mathcal{P}=$ $\left\{M_{1}, \ldots, M_{m}\right\}$ um índice $\left\{1, \ldots, K_{n}^{\infty}\right\}$ representando uma mensagem clássica. A função de decodificação possui a seguinte propriedade:

$$
\operatorname{Pr}\left(g(Y=y) \neq i \mid X^{n}=X^{n}(i)\right)=0 \forall i \in\left\{1, \ldots, K_{n}^{\infty}\right\} .
$$

A taxa do código da Def. 2 é ainda $R=\frac{1}{n} \log K_{n}^{\infty}$. A diferença fundamental entre tal código e o definido na

\footnotetext{
${ }^{1}$ No entanto, o uso de estados entrelaçados entre vários usos do canal é mostrado aumentar a capacidade HSW de canais quânticos com memória [16].
} 
Seç. II é que aqui os elementos do POVM $\mathcal{P}$ são matrizes $M_{k}$ num espaço de Hilbert de dimensão $d^{n}$. A definição da capacidade é essencialmente a mesma da Def. 1, exceto pelo uso do protocolo e do código acima descritos. Para evitar confusão, a capacidade erro-zero para o caso em que medições entrelaçadas são permitidas será denotada por $C_{\infty}^{(0)}(\mathcal{E})$. Visto que o protocolo de $C^{(0)}(\mathcal{E})$ emprega medições individuais, é de se esperar que

$$
C_{\infty}^{(0)}(\mathcal{E}) \geq C^{(0)}(\mathcal{E})
$$

Antes de mostrar a desigualdade na Eq. (10), serão tecidas algumas considerações acerca da ortogonalidade/nãoadjacência dos estados quânticos em $\mathcal{S}$. Na Seç. II, a adjacência entre estados quânticos em $\mathcal{S}$ foi definida com relação a um determinado POVM $\mathcal{P}$. Dois estados $\rho_{i}, \rho_{j} \in \mathcal{S}$ foram ditos ser não-adjacentes com relação a $\mathcal{P}$ se fosse possível distinguir perfeitamente entre $\mathcal{E}\left(\rho_{i}\right)$ e $\mathcal{E}\left(\rho_{j}\right)$ usando $\mathcal{P}$. Dos fundamentos da mecânica quântica [13], é sabido que dois estados quânticos são perfeitamente distinguíveis se, e somente se, eles pertencem a subespaços de Hilbert ortogonais. Isto implica dizer que é possível definir uma relação de adjacência entre estados em $\mathcal{S}$ independentemente da escolha do POVM $\mathcal{P}$ : dois estados quânticos $\rho_{i}, \rho_{j} \in \mathcal{S}$ são ditos não-adjacentes se, e somente se, o subespaço de Hilbert gerado pelos autovetores no suporte de $\mathcal{E}\left(\rho_{i}\right)$ é ortogonal ao subespaço de Hilbert gerado pelos autovetores no suporte de $\mathcal{E}\left(\rho_{j}\right)$. Neste caso denota-se $\rho_{i} \pm_{\mathcal{E}} \rho_{j}$. A notação $\pm_{\mathcal{E}}$ é para lembrar que a ortogonalidade é com relação aos estados na saída do canal quântico $\mathcal{E}$. A mesma noção pode ser aplicada a um estado produto tensorial. Seja $\mathcal{S}=\left\{\rho_{i}, \ldots, \rho_{l}\right\}$ um subconjunto finito de estados quânticos de entrada para $\mathcal{E}$. Considere todas as seqüências de produtos tensoriais de comprimento $n, \mathcal{S}^{\otimes n}$. Duas seqüências $\hat{\rho}_{i}=\rho_{i_{1}} \otimes \cdots \otimes \rho_{i_{n}}$ e $\hat{\rho}_{j}=\rho_{j_{1}} \otimes \cdots \otimes \rho_{j_{n}}$ são ditas ser não-adjacentes se, e somente se, os subespaços de Hilbert gerados pelos autovetores nos suportes de $\mathcal{E}\left(\hat{\rho}_{i}\right)$ e $\mathcal{E}\left(\hat{\rho}_{j}\right)$ são ortogonais. Novamente, denota-se $\hat{\rho}_{i} \pm_{\mathcal{E}} \hat{\rho}_{j}$.

Para um determinado canal quântico $\mathcal{E}$, escolha um subconjunto $\mathcal{S}=\left\{\rho_{i}, \ldots, \rho_{l}\right\}$ de estados quânticos e considere seqüências de comprimento $n, \mathcal{S}^{\otimes n}$. Fazendo uso de tais seqüências, é fácil verificar que o número máximo de mensagens clássicas que se pode transmitir sem erro através de $\mathcal{E}$ é igual ao número máximo de seqüências $\bar{\rho}_{i} \in \mathcal{S}^{\otimes n}$ que são duas-a-duas não-adjacentes. Supondo que existem $K_{n}^{\infty}$ dessas seqüências, a capacidade erro-zero do canal quântico é dada por

$$
C_{\infty}^{(0)}(\mathcal{E})=\sup _{\mathcal{S}} \sup _{n} \frac{1}{n} \log K_{n}^{\infty}
$$

Suponha que a capacidade seja alcançada para um determinado subconjunto $\mathcal{S}$ e um comprimento de código $n$. Isto implica na existência de um código de erro-zero quântico contendo $K_{n}^{\infty}$ palavras-código

$$
\bar{\rho}_{1}, \bar{\rho}_{2}, \ldots, \bar{\rho}_{K_{n}^{\infty}} ; \bar{\rho}_{i} \pm_{\mathcal{E}} \bar{\rho}_{j}, i \neq j
$$

Em outras palavras, os subespaços de Hilbert gerados pelos autovetores no suporte de cada

$$
\begin{aligned}
\mathcal{E}\left(\bar{\rho}_{1}\right) & =\underbrace{\mathcal{E}\left(\rho_{1_{1}}\right) \otimes \mathcal{E}\left(\rho_{1_{2}}\right) \otimes \cdots \otimes \mathcal{E}\left(\rho_{1_{n}}\right)}_{P_{1}} \\
\mathcal{E}\left(\bar{\rho}_{2}\right) & =\underbrace{\mathcal{E}\left(\rho_{2_{1}}\right) \otimes \mathcal{E}\left(\rho_{2_{2}}\right) \otimes \cdots \otimes \mathcal{E}\left(\rho_{2_{n}}\right)}_{P_{2}} \\
& \vdots \\
\mathcal{E}\left(\bar{\rho}_{K_{n}^{\infty}}\right)= & \underbrace{\mathcal{E}\left(\rho_{K_{n_{1}}^{\infty}}\right) \otimes \mathcal{E}\left(\rho_{K_{n_{2}}^{\infty}}\right) \otimes \cdots \otimes \mathcal{E}\left(\rho_{K_{n_{n}}}\right)}_{P_{K_{n}}^{\infty}}
\end{aligned}
$$

são mutuamente ortogonais, em que $P_{i}$ denota o projetor sobre o subespaço de Hilbert gerado pelos autovetores no suporte de $\mathcal{E}\left(\bar{\rho}_{i}\right)$. É fácil verificar que estes estados são completamente distinguíveis na saída do canal. Ainda, uma medição de von Neumann permitindo a distinção é dada por

$$
\mathbb{P}=\left\{P_{1}, \ldots, P_{K_{n}^{\infty}}, P_{K_{n}^{\infty}+1}\right\},
$$

em que $P_{K_{n}^{\infty}+1}=11-\sum_{i=1}^{K_{n}^{\infty}} P_{i}$.

A diferença na definição das capacidades $C_{\infty}^{(0)}(\mathcal{E})$ e $C^{(0)}(\mathcal{E})$ aparece de forma clara quando o conjunto de Eqs. (12) é observado. Claramente vê-se que as medições que permitem alcançar a capacidade devem ser definidas num espaço de Hilbert de dimensão $d^{n}$. No caso do protocolo de $C^{(0)}(\mathcal{E})$ descrito na Seç. II, as medições simultâneas em $n$ estados de saída estão limitadas a

$$
\mathbb{P}^{\prime}=\mathcal{P}^{\otimes n},
$$

em que $\mathcal{P}$ é um POVM cujos operadores pertencem a um espaço de Hilbert de dimensão $d$.

$\mathrm{O}$ resultado a seguir permite verificar a desigualdade na Eq. (10).

Proposição 1 Seja $\mathcal{S}$ um subconjunto de estados quânticos de entrada para $\mathcal{E}$ e $\hat{\rho}_{i}, \hat{\rho}_{j} \in \mathcal{S}^{\otimes n}$. Então $\hat{\rho}_{i} \pm_{\mathcal{E}} \hat{\rho}_{j}$ se, e somente se, existir pelo menos um $1 \leq k \leq n$ tal que $\rho_{i_{k}} \pm_{\mathcal{E}} \rho_{j_{k}}$.

Demonstração: A não-adjacência $\hat{\rho}_{i} \pm_{\mathcal{E}} \hat{\rho}_{j}$ implica que

$$
\begin{aligned}
\operatorname{tr}\left[\mathcal{E}\left(\hat{\rho}_{i}\right) \mathcal{E}\left(\hat{\rho}_{j}\right)\right] & =\operatorname{tr}\left[\left(\bigotimes_{k=1}^{n} \mathcal{E}\left(\rho_{i_{k}}\right)\right)\left(\bigotimes_{k=1}^{n} \mathcal{E}\left(\rho_{j_{k}}\right)\right)\right] \\
& =\prod_{k=1}^{n} \operatorname{tr}\left[\mathcal{E}\left(\rho_{i_{k}}\right) \mathcal{E}\left(\rho_{j_{k}}\right)\right] \\
& =0
\end{aligned}
$$

se, e somente se, $\rho_{i_{k}} \pm_{\mathcal{E}} \rho_{j_{k}}$ para ao menos um $1 \leq k \leq n$. A prova do inverso é trivial.

O resultado da Prop. 1 é ilustrado na Fig. 2. Essencialmente, a proposição afirma que duas seqüências de $n$ produtos tensoriais em $\mathcal{S}$ são não-adjacentes (e portanto completamente distinguíveis na saída do canal) se, e somente se, existir pelo menos uma posição $k$ nas seqüências tal que os esta$\operatorname{dos} \rho_{i_{k}}, \rho_{j_{k}} \in \mathcal{S}$ são distinguíveis. Em outras palavras, a distinguibilidade de quaisquer duas seqüências de $n$ produtos tensoriais de $\mathcal{S}$ na saída do canal quântico depende somente das relações de não-adjacência dos estados quânticos em $\mathcal{S}$. 


$$
\begin{aligned}
& \mathcal{E}\left(\bar{\rho}_{i}\right)=\mathcal{E}\left(\rho_{i_{1}}\right) \otimes \cdots \otimes\left(\mathcal{E}\left(\rho_{i_{k}}\right)\right. \\
& \mathcal{E}\left(\bar{\rho}_{j}\right)=\mathcal{E}\left(\rho_{j_{1}}\right) \otimes \cdots \otimes \otimes \mathcal{E}\left(\rho_{i_{n}}\right) \\
& \mathcal{E}\left(\rho_{j_{k}}\right)
\end{aligned} \otimes \cdots \otimes \mathcal{E}\left(\rho_{j_{n}}\right)
$$

Fig. 2. Duas seqüências de $n$ produtos tensoriais de estados quânticos $\mathcal{S}$ que são não-adjacentes.

Para provar a desigualdade na Eq. (10) é necessário mostrar que $C_{\infty}^{(0)}(\mathcal{E})$ nem sempre pode ser alcançada usando medições individuais.

A distinguibilidade de estados quânticos é uma área bastante estudada em teoria da informação quântica. Uma de suas vertentes consiste na distinção de estados quânticos ortogonais e multi-particionados (multipartite), em que cada parte do sistema quântico está fisicamente separada [17], [18]. Neste caso, o problema consiste em decidir sobre a ortogonalidade dos estados produtos tensoriais através da realização de medições locais e comunicação clássica entre as partes. Este problema, por sua vez, pode ser abordado de duas formas: os estados multi-particionados são entrelaçados [18] ou são produtos tensoriais como os estados da Eq. (12) [17]. Bennett et. al. [19] mostraram a existência de um conjunto de estados quânticos ortogonais, produtos tensoriais de duas partículas de três estados, em que quaisquer dois estados do conjunto, embora globalmente ortogonais, não podem ser distinguidos usando pares de medições individuais, mesmo se os observadores são autorizados a realizar qualquer tipo de operação local e comunicação clássica.

Retornando ao problema de distinguir perfeitamente entre as sequiências da Eq. (12), se medições individuais forem consideradas, fica claro que o protocolo de medição empregado é um caso particular do protocolo do exemplo de Bennett et. al., em que além da restrição de medições individuais impõe-se ainda o fato de que estados do produto tensorial devem ser medidos usando um mesmo POVM $\mathcal{P}$. Isto deve-se ao fato de que, embora quaisquer duas palavras-código na saída do canal, digamos $\mathcal{E}\left(\bar{\rho}_{i}\right)$ e $\mathcal{E}\left(\bar{\rho}_{j}\right)$, sejam ortogonais, os estados $\mathcal{E}\left(\rho_{i_{k^{\prime}}}\right)$ e $\mathcal{E}\left(\rho_{j_{k^{\prime}}}\right)$ não necessariamente os são, visto que a Prop. 1 obriga a ortogonalidade em pelo menos uma posição $k$ mas não em todas.

Dessa forma, fica evidente que nem sempre é possível distinguir perfeitamente entre estados de um conjunto de seqüências de estados quânticos ortogonais usando medições individuais. Portanto, a capacidade erro-zero usando medições coletivas pode ser maior do que a capacidade erro-zero como definida na Ref. [5] e a Eq. (10) é assim verificada.

\section{CAPACIDADE ERRO-ZERO COM ESTADOS QUÂNTICOS NÃO-ORTOGONAIS}

Será discutido nesta seção um exemplo de um canal quântico cuja capacidade erro-zero quântica é calculada de maneira não trivial. $O$ termo não trivial quer dizer que o supremo na Eq. (3) é alcançado para $n>1$ e que subconjuntos $\mathcal{S}$ que alcançam o supremo contenham estados quânticos não ortogonais entre si. O canal quântico do exemplo a seguir não possui interpretação física, embora ele corresponda a algum processo físico. Além da não trivialidade no cálculo da capacidade, este canal dá origem ao pentágono como grafo característico para o subconjunto $\mathcal{S}$ que atinge a capacidade.

Seja $\mathcal{E}$ o canal quântico cujos operadores de Kraus $\left\{E_{1}, E_{2}, E_{3}\right\}$ são dados por

$$
\begin{aligned}
& E_{1}=\left[\begin{array}{ccccc}
0.5 & 0 & 0 & 0 & \frac{\sqrt{49902}}{620} \\
0.5 & -0.5 & 0 & 0 & 0 \\
0 & 0.5 & -0.5 & 0 & 0 \\
0 & 0 & 0.5 & -\frac{\sqrt{457}}{50} & \frac{\sqrt{457}}{50} \\
0 & 0 & 0 & -0.62 & -\frac{289}{1550}
\end{array}\right] \\
& E_{2}=\left[\begin{array}{ccccc}
0.5 & 0 & 0 & 0 & -\frac{\sqrt{49902}}{620} \\
0.5 & 0.5 & 0 & 0 & 0 \\
0 & 0.5 & 0.5 & 0 & 0 \\
0 & 0 & 0.5 & \frac{\sqrt{457}}{50} & -\frac{\sqrt{457}}{50} \\
0 & 0 & 0 & 0.5 & 0.5
\end{array}\right], \quad E_{3}=0.3|4\rangle\langle 4|
\end{aligned}
$$

em que a base computacional para o espaço de Hilbert de dimensão 5 é denotada por $\beta=\{|0\rangle, \ldots,|4\rangle\}$. É fácil verificar que $\sum_{a=1}^{3} E_{a}^{\dagger} E_{a}=\mathbb{1}$, significando que $\mathcal{E} \equiv\left\{E_{a}\right\}$ é um operador linear, positivo e que preserva o traço, de forma a representar um determinado processo físico.

O canal $\mathcal{E}$ foi obtido usando a ferramenta MATLAB, onde foi a imposta a restrição de que $\mathcal{E}$ deveria possuir no máximo dois estados mutuamente não-adjacentes.

Neste caso, a capacidade erro-zero do canal é alcançada pelo subconjunto

$\mathcal{S}=\left\{\left|v_{1}\right\rangle=|0\rangle,\left|v_{2}\right\rangle=|1\rangle,\left|v_{3}\right\rangle=|2\rangle,\left|v_{4}\right\rangle=|3\rangle,\left|v_{5}\right\rangle=\frac{|3\rangle+|4\rangle}{\sqrt{2}}\right\}$.

O conjunto $\mathcal{S}$ alcança o supremo na Eq. (5) pelo seguinte motivo. Dentre os grafos característicos com até 5 vértices e tendo número de clique menor ou igual a dois, o grafo que fornece a maior capacidade é o pentágono [6]. É fácil verificar que o conjunto $\mathcal{S}$ dá origem ao pentágono como grafo de adjacência. Para isso, basta explicitar as relações de nãoadjacência entre os estados de $\mathcal{S}$ :

$$
\begin{gathered}
\left|v_{1}\right\rangle \pm_{\mathcal{E}}\left|v_{3}\right\rangle \quad\left|v_{1}\right\rangle \pm_{\mathcal{E}}\left|v_{4}\right\rangle \quad\left|v_{2}\right\rangle \pm_{\mathcal{E}}\left|v_{4}\right\rangle \\
\left|v_{2}\right\rangle \pm_{\mathcal{E}}\left|v_{5}\right\rangle \quad\left|v_{3}\right\rangle \pm_{\mathcal{E}}\left|v_{5}\right\rangle .
\end{gathered}
$$

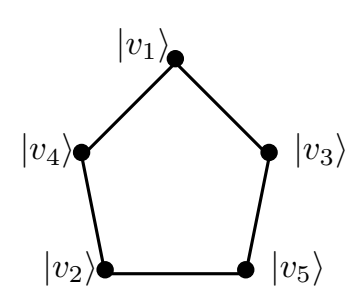

(a)

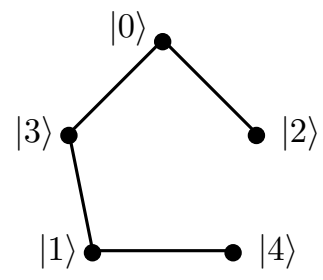

(b)
Fig. 3. (a) Grafo característico $\mathcal{G}$ para o subconjunto $\mathcal{S}$ que alcança a capacidade erro-zero. (b) Grafo característico para o caso em que o estado $\left|v_{5}\right\rangle \in \mathcal{S}$ é substituído pelo estado $|4\rangle$.

O grafo característico é mostrado na Fig. 3(a). Note que no caso em que as palavras-código são de comprimento um só é possível transmitir no máximo duas mensagens clássicas sem erro através do canal quântico, por exemplo, escolhendo $\left|v_{1}\right\rangle$ e $\left|v_{3}\right\rangle$ ou $\left|v_{2}\right\rangle$ e $\left|v_{4}\right\rangle$. Entretanto, seguindo a construção 
inicialmente feita por Shannon [6], é possível construir um código contendo cinco palavras-código não adjacentes:

$$
\begin{array}{r}
\bar{\rho}_{1}=\left|v_{1}\right\rangle\left|v_{1}\right\rangle, \quad \bar{\rho}_{2}=\left|v_{2}\right\rangle\left|v_{3}\right\rangle, \quad \bar{\rho}_{3}=\left|v_{3}\right\rangle\left|v_{5}\right\rangle \\
\bar{\rho}_{4}=\left|v_{4}\right\rangle\left|v_{2}\right\rangle, \quad \bar{\rho}_{5}=\left|v_{5}\right\rangle\left|v_{4}\right\rangle .
\end{array}
$$

A capacidade do pentágono foi determinada por Lovász [20], e é alcançada quando o canal é usado duas ou mais vezes. Logo, a capacidade erro-zero do canal quântico $\mathcal{E}$ é dada por:

$$
C_{\infty}^{(0)}(\text { pentagon })=\frac{1}{2} \log 5 \approx 1,16 \text { bits por uso. }
$$

Portanto, $C_{\infty}^{(0)}$ (pentagon) é a máxima taxa em que informação clássica pode ser transmitida através do canal $\mathcal{E}$ com uma probabilidade de erro igual a zero.

É interessante notar que se ao invés de $\mathcal{S}$ fosse escolhida a base computacional acima, então uma adjacência entre os $|2\rangle$ e $|4\rangle$ é verificada, de forma que o grafo característico para $\beta$ é mostrado na Figura 3(b). Claramente este grafo possui capacidade igual a um e portanto inferior à capacidade do pentágono.

\section{CONCLUSÕES}

$\mathrm{Na}$ forma em que foi inicialmente definida, a capacidade erro-zero de canais quânticos previa o uso de medições individuais na saída do canal quântico. Neste artigo foi verificado o comportamento do valor da capacidade para o caso em que medições entrelaçadas entre várias saídas do canal são permitidas. Foi mostrado que tais medições podem aumentar a capacidade erro-zero dos canais quânticos. A razão para tal reside no fato de que todas as palavras-código de um código de bloco de erro-zero devem ser completamente distinguíveis na saída do canal, e esta distinguibilidade depende somente da distinguibilidade dos estados quânticos do alfabeto do código, como demonstrado na Prop. 1. Entretanto, Bennett et. al. mostraram que nem sempre é possível distinguir estados produtos tensoriais de um conjunto de estados quânticos ortogonais usando medições individuais. Foi observado também que não é necessária uma maximização sobre as medições para o cálculo da capacidade com medições coletivas.

Finalmente, foi mostrado um exemplo de um canal quântico cuja capacidade é não trivialmente calculada, ou seja, a capacidade é alcançada por um conjunto de estados quânticos não ortogonais e o canal requer duas ou mais utilizações para que a capacidade $C_{\infty}^{(0)}(\mathcal{E})$ seja atingida.

\section{Agradecimentos}

Os autores gostariam de agradecer o Programa Al $\beta$ an, Programa de bolsas de alto nível da União Europeia para América Latina, bolsa $\mathrm{n}^{\circ}$ E05D051893BR, bem como o CNPq (CTINFO Quanta, contrato \# 552254/02-9), pelo apoio financeiro. Este trabalho foi fincanciado em parte pelo projeto Europeu SECOQC (contrato \# IST-2003-506813).

\section{REFERÊNCIAS}

[1] B. Schumacher and M. D. Westmoreland. Sending classical information via noisy quantum channels. Phys. Rev. A, 56(1):131-138, 1997.

[2] A. S. Holevo. The capacity of the quantum channel with general signal states. IEEE Trans. Info. Theory, 44(1):269-273, 1998.

[3] C. H. Bennett, P. W. Shor, J. A. Smolin, and A. V. Thapliyal. Entanglement-assisted classical capacity of noisy quantum channels. Phys. Rev. Lett., 83:3081-3084, 1999.

[4] P. W. Shor. The adaptive classical capacity of a quantum channel, or information capacities of three symmetric pure states in three dimensions. IBM. J. Res. \& Dev., 48(1):115-137, 2004.

[5] R. A. C. Medeiros and F. M. de Assis. Quantum zero-error capacity. Int. J. Quant. Inf., 3(1):135-139, 2005.

[6] C. E. Shannon. The zero error capaciy of a noisy channel. IRE Trans. Inform. Theory, IT-2(3):8-19, 1956.

[7] J. Köner and A. Orlitsky. Zero-error information theory. IEEE Trans. Info. Theory, 44(6):2207-2229, 1998.

[8] R. A. C. Medeiros and F. M. de Assis. Capacidade erro-zero de canais quânticos e estados puros. In Anais do XXII Simpósio Brasileiro de Telecomunicações, XXII Simpósio Brasileiro de Telecomunicações SBrT'05, Campinas-PB, Brazil, 2005.

[9] R. A. C. Medeiros, R. Alléaume, F. M. de Assis, and G. Cohen. Quantum state characterization for the zero-error capacity. In Proceeding of the IEEE Information Theory Winter School 2007, page 19, France, 2007.

[10] R. A. C. Medeiros and F. M. de Assis. Quantum zero-error capacity and HSW capacity. In Proceedings of the The Seventh International Conference on Quantum Communication, Measurement and Computing QCMC'04, volume 734 of AIP Conference Proceedings, pages 52-54. American Institute of Physics, 2004.

[11] R. A. C. Medeiros, R. Alléaume, G. Cohen, and F. M. de Assis. Zeroerror capacity of quantum channel and noiseless subsystems. In IEEE International Telecommunications Symposium ITS2006, Brazil, 2006.

[12] R. A. C. Medeiros, R. Alléaume, G. Cohen, and F. M. de Assis. quant$\mathrm{ph} / 0611042$.

[13] M. A. Nielsen and I. L. Chuang. Quantum Computation and Quantum Information. Cambridge University Press, Cambridge, 2000.

[14] R. A. C. Medeiros and F. M. de Assis. Zero-error capacity of a quantum channel. In Proceedings of the 11th International Conference on Telecommunications, volume 3124 of Lecture Notes in Computer Science, pages 100-105, Heidelberg, 2004. Springer-Verlag Heidelberg.

[15] B. Bollobás. Modern graph theory. Springer-Verlag New York, Inc., New York, 1998.

[16] C Macchiavello and G. M. Palma. Entanglement-enhanced information transmission over a quantum channel with correlated noise. Phys. Rev. A, 65(5):050301, Apr 2002.

[17] W. K. Wootters. Distinguishing unentangled states with an unentangled measurement. quant-ph/0506149, 2005.

[18] J. Walgate, A. J. Short, L. Hardy, and V. Vedral. Local distinguishability of multipartite orthogonal quantum states. quant-ph/0007098, 2000.

[19] Charles H. Bennett, David P. DiVincenzo, Christopher A. Fuchs, Tal Mor, Eric Rains, Peter W. Shor, John A. Smolin, and William K. Wootters. Quantum nonlocality without entanglement. Phys. Rev. A, 59(2):1070-1091, Feb 1999.

[20] L. Lovász. On the Shannon capacity of a graph. IEEE Trans. Info. Theory, 25(1):1-7, 1979. 